\title{
Radio halos in merging clusters of galaxies
}

\author{
S. Giacintucci ${ }^{1,2}$, T. Venturi ${ }^{3}$, S. Bardelli ${ }^{2}$, G. Brunetti ${ }^{3}$, \\ D. Dallacasa ${ }^{1,3}$, P. Rao ${ }^{4}$ and E. Zucca ${ }^{2}$ \\ ${ }^{1}$ Dipartimento di Astronomia, Univ. Bologna, via Ranzani 1, I-40127 Bologna, Italy \\ ${ }^{2}$ Osservatorio astronomico, via Ranzani 1, I-40127 Bologna, Italy \\ ${ }^{3}$ Istituto di Radioastronomia - CNR, via Gobetti 101, I-40129 Bologna, Italy \\ ${ }^{4}$ National Centre for Radio Astrophysics, Pune University Campus, India
}

\begin{abstract}
We present the preliminary results of $235 \mathrm{MHz}, 327 \mathrm{MHz}$ and $610 \mathrm{MHz}$ observations of the galaxy cluster A3562 in the core of the Shapley Concentration (SC). The purpose of these observations, carried out with the Giant Metrewave Radio Telescope (GMRT, Pune, India) was to study the radio halo located at the centre of A3562 and determine the shape of its radio spectrum at low frequencies, in order to understand the origin of this source. In the framework of the re-acceleration model, the preliminary analysis of the halo spectrum suggests that we are observing a young source (few $10^{8} \mathrm{yrs}$ ) at the beginning of the re-acceleration phase.
\end{abstract}

\section{Introduction}

A number of X-ray luminous galaxy clusters show large-scale synchrotron radio emission associated to the intracluster medium. These diffuse sources are know as radio halos, when they are located at the centre of the hosting cluster and show low or negligible polarization, and radio relics, when found in the cluster outskirts and highly polarized.

Both halos and relics have low surface brightness, large linear size (from $\sim 0.5 \mathrm{Mpc}$ to more than $1 \mathrm{Mpc})$ and steep integrated spectra, i.e. $\alpha>1\left(\mathrm{~S} \propto \nu^{-\alpha}\right)$.

Radio halos and relics represent the most striking evidence for $\mu \mathrm{G}$ magnetic fields on cluster scale and relativistic electrons diffused within the whole cluster volume (for a recent review see Giovannini \& Feretti 2002).

The existence of this class of radio sources is believed to be connected to cluster mergers, since thus far they have only been found in clusters with significant signs of a current or recent merging event. In particular the leading hypothesis for the origin of the observed radio emission from these objects is a re-acceleration process, probably via turbolence powered by cluster mergers, of a population of relatively low energy $\left(\gamma \sim 10^{3}\right)$ electrons, initially injected in the intracluster medium (two-phase model, Brunetti et al. 2001).

\section{The radio halo in $\mathbf{A} 3562$}

The cluster A3562 is the easternmost component of the A3558 complex, a chain of three clusters and two SC groups which form a single physically connected structure, whose complex dynamical stage of merging is supported by a large amount of observational data in all bands (see for instance Ettori et al. 1997, Ettori et al. 2000).

The existence of a radio halo at the A3562 centre was confirmed by deep radio observations at $1.4 \mathrm{GHz}$ carried out with VLA (Venturi et al. 2003).

In the framework of the re-acceleration model, the halo in A3562 seems to play a very special role, since its largest linear size (LLS $\sim 600 \mathrm{kpc}$ ) and $1.4 \mathrm{GHz}$ radio power 


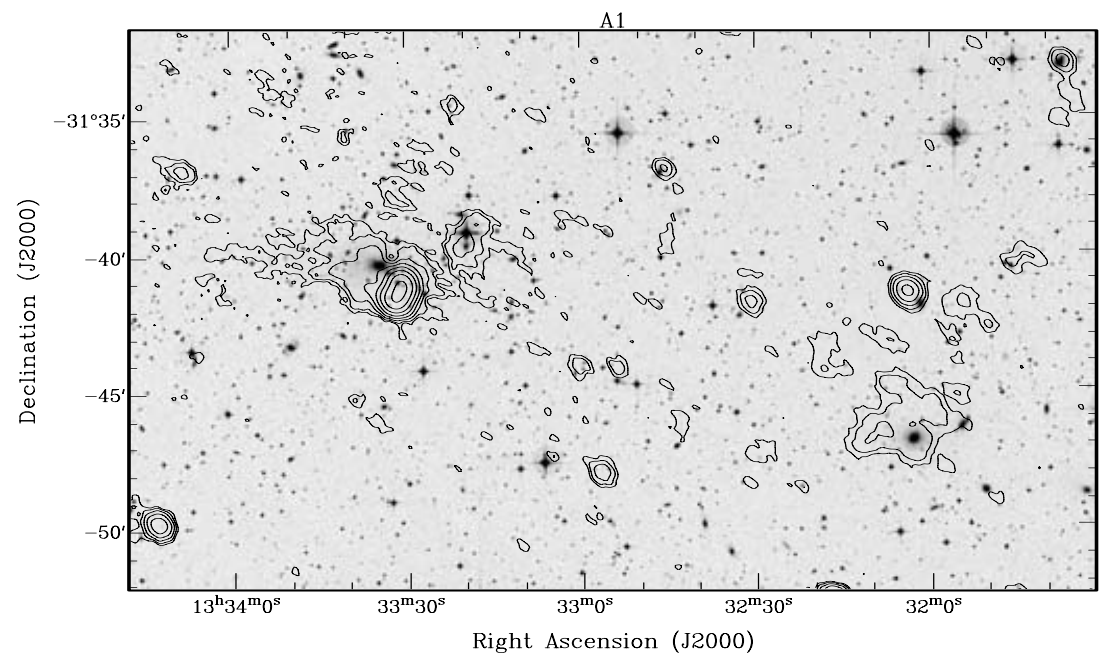

Figure 1. $327 \mathrm{MHz}$ GMRT image of the radio halo in A3562 and the south-western extended emission in the direction of the SC 1329-313 group, overlaid on the optical DSS-2 image. The restoring beam is $41.98^{\prime \prime} \times 35.13^{\prime \prime}$. The rms in the image is $\sim 0.25 \mathrm{mJy} /$ beam and the lowest contour is $0.8 \mathrm{mJy} /$ beam.

$\left(\mathrm{P}_{1.4 G H z}=2.14 \times 10^{23} \mathrm{~W} \mathrm{~Hz}^{-1}\right)$ are among the lowest values found for this class of radio sources.

The halo nicely fits the correlations existing between the halo radio power and the cluster X-ray luminosity and temperature (Bacchi et al. 2003), extending them to lower values for all quantities involved.

According to the two-phase model, the halo in A3562 could be interpreted either as the result of a low efficiency re-acceleration process or as a young source at the beginning of the re-acceleration phase. It has been established that the head-tail radio galaxy J1333-3141, located within the halo emission, has deposited in the intracluster medium a number of electrons high enough to feed the halo if it has been active for a considerable fraction of its crossing time ( $\mathrm{t}_{\text {cross }} \sim 6 \times 10^{8} \mathrm{yrs}$ ). This result seems to suggest that the halo at the centre of A3562 is young as compared to the other radio halos known to date (Venturi et al. 2003). However only low frequencies observations allow to discriminate between the two possibilities.

\section{Low frequency observations of the halo in A3562}

With the purpose of studing the morphology of the halo in A3562 and determine the shape of its synchrotron spectrum at low frequencies, we carried out observations at $235 \mathrm{MHz}, 327 \mathrm{MHz}$ and $610 \mathrm{MHz}$ with the Giant Metrewave Radio Telescope (GMRT, Pune, India). Thanks to the $\mathrm{u}-\mathrm{v}$ coverage of this instrument, a range of resolutions at all frequencies (from few arcsec to tens of arcsec) is allowed. This ensures an accurate determination of the total radio flux density of the halo, after a careful subtraction of the individual point sources embedded in the halo emission.

In Figure 1 we report the image of the radio halo in A3562 at $327 \mathrm{MHz}$ overlaid on the optical DSS-2 image, and in Figure 2 we present the $610 \mathrm{MHz}$ image of the halo overlaid on the $\mathrm{X}$-ray XMM image. 


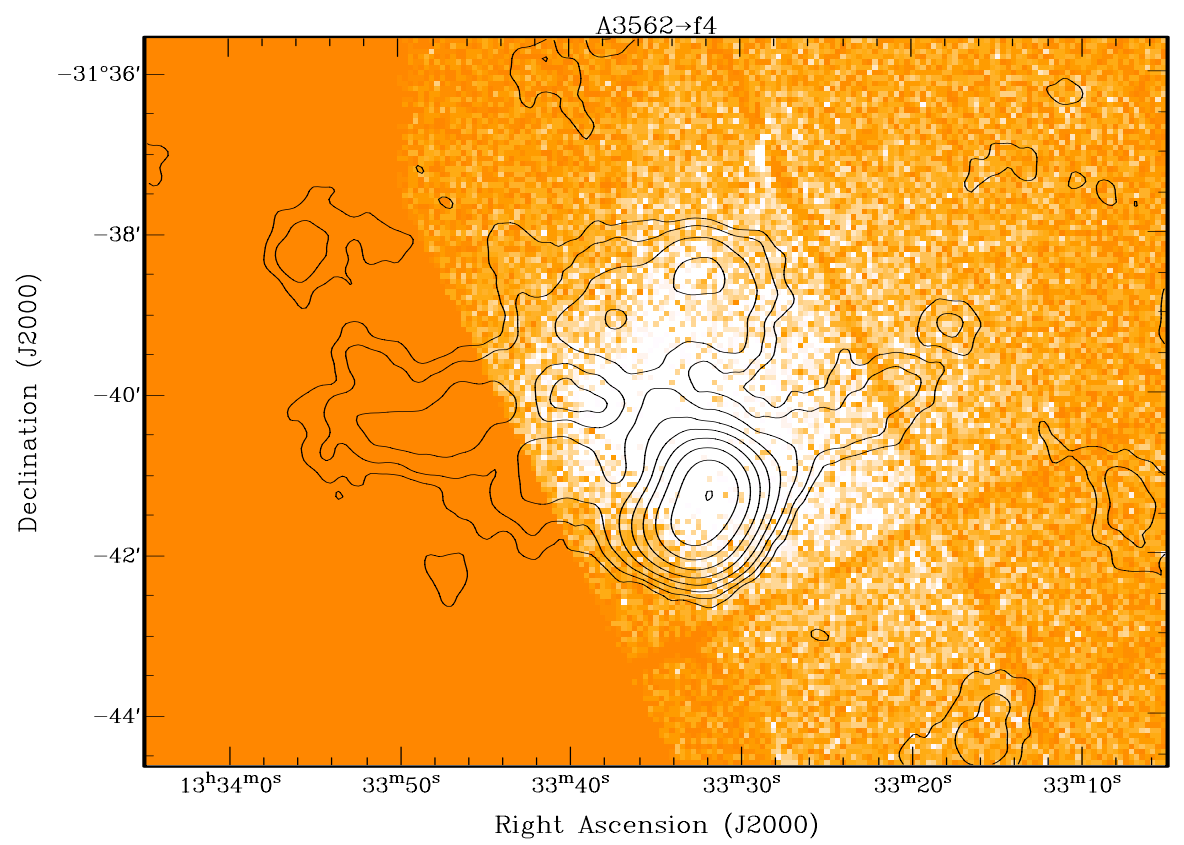

Figure 2. $610 \mathrm{MHz}$ GMRT image of the radio halo in A3562 overlaid on the X-ray XMM image. The restoring beam is $41.98^{\prime \prime} \times 35.13^{\prime \prime}$. The $\mathrm{rms}$ in the image is $\sim 0.15 \mathrm{mJy} /$ beam and the lowest contour is $0.45 \mathrm{mJy} /$ beam.

An extended radio emission, located south-west of the halo, surrounds the radio source J1332-3146 (Figure 1), identified with the brightest galaxy in the SC 1329-313 group (Giacintucci et al. 2003). The nuclear component, detected at $1.4 \mathrm{GHz}$, disappears at low frequency. This seems to rule out any connection between the low brightness extended emission and an active nucleus, suggesting that its origin is most likely due to cluster merger re-acceleration.

The preliminary results of our low frequency data suggest that the spectrum of the radio halo is steep up to $\sim 610 \mathrm{MHz}$, with $\alpha_{610 \mathrm{MHz}}^{1.4 \mathrm{~Hz}} \sim 1.9$, and it flattens beyond $610 \mathrm{MHz}$, with $\alpha_{235 M H z}^{610 M H z} \sim 1$. This seems to be in good agreement with a very recent re-acceleration phase, supporting the idea that the radio halo in A3562 is young and that its existence is strongly connected to the ongoing major merger in the A3558 complex (Venturi et al. in preparation).

\section{References}

Bacchi, M. Feretti, L. Giovannini, G. Govoni, F. 2003 AESA in press (astro-ph/0301296).

Brunetti, G. Setti, G. Feretti, L. Giovannini, G. 2001 MNRAS 320, 365.

Ettori, S. Fabian, A.C. White, D.A. 1997 MNRAS 289, 787.

Ettori, S. Bardelli, S. De Grandi, S. Molendi, S., Zamorani, G. Zucca, E. 2000 MNRAS 318, 239.

Giacintucci, S. Venturi, T., Bardelli, S. Dallacasa, D. Zucca, E. 2003 A\&A in press (astro$\mathrm{ph} / 0311251)$.

Giovannini, G. \& Feretti, L. 2002 Astroph. Sp. Sci. Lib. 272, 197.

Venturi, T. Bardelli, S. Dallacasa, D. Brunetti, G. Giacintucci, S. Hunstead, R.W., Morganti, R. $2003 A \& A$ 402, 913. 